\title{
Heparin and protamine interaction
}

Gilbert Blaise MD

$\mathrm{I}$ $\mathrm{N}$ this issue of the Canadian Journal of Anaesthesia Komatsu et al. discuss one of the mechanisms producing haemodynamic effects in the protamine-heparin reaction. ${ }^{1}$

The reversal of heparinization by protamine may produce life-threatening haemodynamic disturbances. These may occur not only after cardiac surgery when the large amounts of heparin given during cardiopulmonary bypass (CPB) are neutralized by an equivalent amount of protamine at a time when the myocardium is recovering from the ischaemic insult, but also after neutralization of heparin given in smaller doses for peripheral vascular surgery or angioplasty. Very often, protamine given after cardiac surgery induces pulmonary hypertension, hypoxaemia and systemic hypotension. The systemic hypotension may be severe: cardiovascular collapse and cardiac arrest have been reported. The mechanism of the physiological disturbances induced by protamine and heparin-protamine complexes is not fully known.

Pulmonary hypertension is due to the secretion of thromboxane $A_{2}$ and 5 ' hydroxytryptamine (serotonin). Hypoxaemia results from ventilation/perfusion mismatching caused by pulmonary oedema secondary to pulmonary hypertension, activation of leucocytes and platelets that accumulate in the lungs, and activation of the classical complement pathway. ${ }^{2}$ Bronchospasm is due to the secretion of histamine and other bronchoactive substances.

Systemic hypotension may also be due to the systemic release of histamine; ${ }^{3}$ in patients allergic to protamine, this could lead to cardiovascular collapse, myocardial depression or right ventricular failure secondary to pulmonary hypertension. Komatsu et al. have shown that protamine is a vasodilator which stimulates nitric oxide (NO) production or action. ${ }^{1}$ They showed that the hypotension, induced by protamine, can be prevented in rats treated with L-NMMA, an L-arginine analogue, which non-specifically inhibits NO synthase (NOS). The same results have been confirmed in vivo by Raikar $e t a l .^{4}$ and ex vivo in isolated aortic and pulmonary ring preparations in which pro- tamine induces an endothelium-dependent relaxation. ${ }^{5}$ Protamine may bind to a protamine receptor or directly stimulate endothelial cells by its polycationic charges. Histamine, released by protamine, may also increase NO production through stimulation of endothelial histaminic receptors. The exact source of NO in patients suffering from protamine-induced hypotension after cardiac surgery is not established as $\mathrm{CPB}$ induces a systemic inflammatory response syndrome which induces a constitutive NOS in endothelial cells and an inducible NOS in vascular smooth muscle, cardiomyocytes and inflammatory cells. There are three different isoforms of NOS and two are always expressed. They are the constitutive ones, eNOS and nNOS, which are mainly found in endothelial cells and neuronal cells respectively. The third NOS is only expressed in some pathological conditions such as the inflammatory process; it is the inducible NOS (iNOS). Constitutive NOS produce picomoles of NO when they are stimulated by chemical or physical stimuli whereas iNOS produces nanomoles of NO in a continuous manner when it is induced.

Hypotension induced by protamine is also due to myocardial depression; ${ }^{4}$ and this too may be induced by NO. Why does protamine induce pulmonary vasoconstriction and systemic vasodilation? Human pulmonary vessels are particularly responsive to thromboxane $\mathrm{A}_{2}$ and serotonin which are potent vasoconstrictors. Following $\mathrm{CPB}$, the pulmonary endothelium is not functional as the receptor-mediated release of NO pathway (which involves the G proteins pathway) is altered, due to reperfusion injury and pulmonary inflammation induced by $C P B$. In the pulmonary circulation, the vasoconstrictive effect of thromboxane $A_{2}$ and serotonin are then insufficiently opposed by the endothelium-dependent relaxing pathway. ${ }^{6}$ The reperfusion injury syndrome also occurs in the myocardium. The effect of protamine on the coronary circulation in the reperfused myocardi$\mathrm{um}$ is not well known. However, it has been shown that protamine decreases venous graft patency after 
coronary artery bypass surgery. ${ }^{7}$ Heparin interferes with the endothelium-dependent vasomotion by stimulating not only the endothelial phospholipase $\mathrm{A}_{2}$ and the production of arachidonic acid and prostacyclin, but also the production of NO as it has been shown in isolated cultured endothelial cells. ${ }^{8}$ Hypotension induced by heparin could then be due to the release of both NO and prostacyclin by endothelial cells.

What is the treatment of the protamine reaction? Pulmonary hypertension and systemic hypotension can be avoided or reduced by slow infusion of protamine through a peripheral vein to optimize the formation of heparin-protamine complexes. Pretreatment with $\mathrm{H}_{2}$ receptor-blockers has been used with success to avoid systemic and bronchial reactions to protamine. Pulmonary hypertension is usually treated with nitrovasodilators such as nitroglycerine and sodium nitroprusside. Systemic hypotension is treated by vasomotor and inotropic support such as catecholamines and phosphodiesterases inhibitors.

Komatsu $e t a l .{ }^{1}$ and Raikar $e t$ al. ${ }^{4}$ both suggested that block of NOS by L-NMMA could decrease the hypotension induced by protamine. The L-NMMA not only reestablished peripheral tone but also improved myocardial contractility but it may also decrease the excessive amount of NO produced by the inflammatory response to $\mathrm{CPB}$ which could affect platelet function and potentiate bleeding. It is worth mentioning that aprotinin, which is used more frequently to reduce bleeding after cardiac surgery, decreases NO production in these patients. ${ }^{9} \mathrm{~L}$ NMMA is currently being studied in an international clinical trial to determine if it decreased mortality in patients suffering from septic shock. The goal of treatment is to counteract the massive production of NO by iNOS induced by different cytokines. One frequent adverse outcome observed during the trial is pulmonary hypertension.

Using L-NMMA in patients after cardiac surgery may induce severe pulmonary hypertension. Blockade of NOS would also affect the reperfusion injury syndrome seen in the lung and the myocardium and probably the systemic inflammatory response syndrome (SIRS) induced by CPB. We do not believe that LNMMA should be used alone. However, simultaneous reduction in NO synthesis and treatment with inhaled NO to prevent or treat pulmonary hypertension may have a place in patient treatment after cardiac surgery. We and others have shown that inhaled NO has some extrapulmonary effects. ${ }^{10}$ Inhaled NO may interfere with cardiac and renal function, inhibit leucocyte adhesion to vascular beds in and outside the pulmonary circulation, and interfere with the reperfusion injury in the lung and myocardium. Further research is necessary to determine the best drug combination to modulate the NO pathway after cardiac surgery.

\section{Interaction de l'héparine et de la protamine}

Dans le présent numéro du Journal canadien d'anesthésie, Komatsu et coll. présentent l'un des mécanismes des effets hémodynamiques induits lors d'une réaction entre la protamine et l'héparine. ${ }^{1}$

La neutralisation de l'héparinisation par la protamine peut produire des troubles hémodynamiques qui mettent en jeu la vie du patient. Ces effets peuvent se produire non seulement après une chirurgie cardiaque, lorsque les grandes quantités d'héparine administrées pendant la circulation extracorporelle (CEC) sont neutralisées par une quantité équivalente de protamine au moment où le myocarde se remet de l'agression ischémique, mais aussi après la neutralisation de l'héparine fournie en petites doses lors d'une chirurgie vasculaire périphérique ou d'une angioplastie. Très souvent, la protamine administrée après la chirurgie cardiaque provoque de l'hypertension pulmonaire, de l'hypoxémie et de l'hypotension générale. L'hypotension générale peut être sévère : collapsus cardiovasculaire et arrêt cardiaque ont été rapportés. On ne connaît pas encore parfaitement le mécanisme des désordres physiologiques causés par la protamine et les complexes d'héparine-protamine.

L'hypertension pulmonaire est causée par la sécrétion de thromboxane $\mathrm{A}_{2}$ et de 5-hydroxytrypta mine (sérotonine). L'hypoxémie résulte d'une perturbation du rapport ventilation/perfusion, causée par l'œdème pulmonaire secondaire à l'hypertension pulmonaire, par l'activation des leucocytes et des plaquettes qui s'accumulent dans les poumons et par l'activation de la voie classique du complément. ${ }^{2}$

L'hypotension générale peut aussi dépendre de la libération d'histamine dans le sang, chez les patients allergiques à la protamine. ${ }^{3}$ Cela peut conduire à un collapsus cardiovasculaire, à une dépression myocardique ou à une insuffisance ventriculaire droite secondaire à l'hypertension pulmonaire. Komatsu et coll. ont montré que la protamine est un vasodilatateur qui stimule la production ou l'action de l'oxyde nitrique (NO). ${ }^{1}$ Ils ont montré que l'on peut prévenir l'hypotension, induite par la protamine, chez des rats traités avec la L- 
NMMA, un analogue de la L-arginine qui inhibe la NO synthase (NOS) de façon non spécifique. Les mêmes résultats ont été obtenus in vivo par Raikar et coll. ${ }^{4}$ et in vitro dans des préparations d'anneaux pulmonaires et d'anneaux aortiques isolés dans lesquels la protamine provoque une dilatation dépendante de l'endothélium. ${ }^{5}$ La protamine peut se fixer à un récepteur de protamine ou stimuler directement les cellules endothéliales de ses charges polycationiques. L'histamine, libérée par la protamine, peut aussi accroitre la production de NO en stimulant les récepteurs d'histamine de l'endothélium. La source exacte de NO chez les patients souffrant d'hypotension induite par la protamine, après une chirurgie cardiaque, n'a pas été identifiée, car la CEC provoque un syndrome de réaction inflammatoire généralisée qui stimule une NOS constitutive dans les cellules endothéliales et produit une NOS inductible dans les fibres lisses de la paroi artérielle, dans les cardiomyocytes et dans les cellules inflammatoires. Il existe trois NOS isomorphes différentes, deux d'entre elles étant toujours exprimées. Ce sont la eNOS et la nNOS constitutives, que l'on retrouve surtout dans les cellules endothéliales et neuronales, respectivement. La troisième NOS se retrouve seulement dans certaines conditions pathologiques comme l'inflammation; c'est la NOS inductible (iNOS). Les NOS constitutives produisent des picomoles de NO quand elles sont stimulées par un agent chimique ou physique alors que la iNOS, quand elle est induite, produit des nanomoles de NO d'une manière continue.

L'hypotension déclenchée par la protamine peut être aggravée aussi par une dépression myocardique ${ }^{4}$, elle- même induite par le NO. Pourquoi la protamine amène-t-elle une vasoconstriction pulmonaire et une vasodilatation générale? Les vaisseaux pulmonaires humains sont particulièrement sensibles au thromboxane $\mathrm{A}_{2}$ et à la sérotonine qui sont de puissants vasoconstricteurs. Après la CEC, l'endothélium pulmonaire n'est plus fonctionnel, la libération de $\mathrm{NO}$, secondaire à la stimulation endothéliale par des agents qui impliquent la participation des protéines $G$, est réduite par la reperfusion et par une inflammation pulmonaire induites par la CEC. Dans la circulation pulmonaire, l'effet vasoconstricteur du thomboxane $\mathrm{A}_{2}$ et de la sérotonine est alors insuffisamment neutralisé par le NO endothélial. ${ }^{6}$ Le syndrome de lésion de reperfusion survient également dans le myocarde. L'effet de la protamine sur la circulation coronarienne dans le myocarde reperfusé est mal connu. Cependant, on a prouvé que la protamine diminue le diamètre de la greffe veineuse après un pontage de l'artère coronaire. ${ }^{7}$ L'héparine entrave la modification du calibre des vaisseaux, qui dépend de l'endothélium, en stimulant non seulement la phospholipase $A_{2}$ endothéliale et la production d'acide arachidonique et de prostacycline, mais aussi en stimulant la production de NO comme on a pu le montrer avec des cellules endothéliales isolées mises en culture. ${ }^{8}$ L'hypotension provoquée par l'héparine peut donc être reliée à la libération autant de NO que de prostacycline par les cellules endothéliales.

Comment traiter la réaction à la protamine? L'hypertension pulmonaire et l'hypotension générale peuvent être évitées ou réduites par une perfusion lente de protamine dans une veine périphérique afin d'optimaliser la formation des complexes héparineprotamine. Le prétraitement avec des inhibiteurs de récepteur $\mathrm{H}_{2}$ a été utilisé avec succès pour éviter des réactions généralisées et bronchiques à la protamine. L'hypertension pulmonaire est normalement traitée avec des nitrovasodilatateurs comme la nitroglycérine et le nitroprussiate de sodium. L'hypotension générale est traitée par une association de substances vasoactives et inotropes comme les catécholamines et les inhibiteurs de phosphodiestérases.

Komatsu et coll. ${ }^{1}$ et Raikar et coll. ${ }^{4}$ suggèrent que le blocage de NOS par la L-NMMA peut diminuer l'hypotension provoquée par la protamine. La L-NMMA non seulement rétablit la tonicité périphérique, mais elle améliore aussi la contractilité myocardique. Toutefois, elle peut également diminuer la quantité excessive de NO produit par la réaction inflammatoire à la $\mathrm{CEC}$, laquelle peut agir sur la fonction plaquettaire et augmenter le saignement. Il est bon de mentionner que l'aprotinine, qui est plus souvent utilisée pour réduire le saignement après une chirurgie cardiaque, réduit la production de NO chez les patients qui ont subi une telle intervention. ${ }^{9} \mathrm{La}$ L-NMMA est présentement à l'étude dans un essai clinique international dans le but de déterminer si elle diminue la mortalité chez les patients souffrant de choc septique. L'objectif du traitement est de contrecarrer la production massive de NO par la iNOS induite par différentes cytokines. Un effet secondaire fréquemment observé pendant l'essai est l'hypertension pulmonaire.

L'emploi, chez des patients, de la L-NMMA, après une chirurgie cardiaque, peut provoquer une hyperten sion pulmonaire sévère. Le blocage de la NOS pourrait aussi affecter le syndrome de lésion de reperfusion dans les poumons et le myocarde et, probablement, le syndrome de réaction inflammatoire généralisée (SRIG) produit par la CEC. Nous ne pensons pas que la L-NMMA devrait être utilisée seule. Cependant, la réduction de la synthèse de NO et le traitement simultané avec inhalation de NO, pour prévenir ou traiter l'hypertension, peuvent faire partie 
du traitement d'un patient après une chirurgie cardiaque. Nous avons montré, avec d'autres, que le NO inhalé a une action extrapulmonaire. ${ }^{10} \mathrm{Il}$ peut interférer avec les fonctions cardiaque et rénale, inhiber l'adhésion des leucocytes aux lits vasculaires à l'intérieur et à l'extérieur de la circulation pulmonaire et agir sur la lésion de reperfusion dans les poumons et le myocarde. D'autres recherches sont nécessaires pour déterminer la meilleure combinaison de médicaments qui pourrait moduler la voie métabolique du NO après une chirurgie cardiaque.

\section{References}

1 Komatsu H, Enzan K, Matsuura S, Kurosawa S, Mitsubata $H$. Systemic hypotensive response to protamine caused by chronic inhibition of nitric oxide synthase in rats. Can J Anaesth 1998; 45: 1186-89.

2 Loubser PG. Effect of methylprednisolone on complement activation during heparin neutralization. J Cardiovasc Pharmacol 1997; 29: 23-7.

3 Kambam J, Meszaros R, Merrill W, Stewart J, Smith $B E$, Bender $H$. Prophylactic administration of histamine $_{1}$ and histamine ${ }_{2}$ receptor blockers in the prevention of protamine-related haemodynamic effects. Can J Anaesth 1990; 37: 420-2.

4 Raikar GV, Hisamochi K, Raikar B-LN, Scbaff HV. Nitric oxide inhibition attenuates systemic hypotension produced by protamine. J Thorac Cardiovasc Surg 1996; 111: 1240-6.

5 Evora PRB, Pearson PJ, Schaff HV. Protamine induces endothelium-dependent vasodilatation of the pulmonary artery. Ann Thorac Surg 1995; 60: 405-10.

6 Morita K, Ibnken K, Buckberg GD, Sherman MP, Ignarro $L J$. Pulmonary vasoconstriction due to impaired nitric oxide production after cardiopulmonary bypass. Ann Thorac Surg 1996; 61: 1775-80.

7 Belboul A, Al-Khaja N. The effect of protamine on the epicardial microflow and the graft flow in open- heart surgery. Perfusion 1997; 12: 99-106.

8 Li J, Hajarizadeh H, La Rosa CA, Robrer MJ, Vander Salm ThJ, Cutler BS. Heparin and protamine stimulate the production of nitric oxide. J Cardiovasc Surg 1996; 37: 445-52.

9 Hill GE, Springall DR, Robbins $R A$. Aprotinin is associated with a decrease in nitric oxide production during cardiopulmonary bypass. Surgery 1997; 121: 449-55.

10 Troncy E, Francceur M, Salazkin I, et al. Extra-pulmonary effects of inhaled nitric oxide in swine with and without phenylephrine. Br J Anaesth 1997; 79: 631-40. 